\title{
Complex Network Theory in Urban Traffic Network
}

\author{
Mengxin $\mathrm{Li}^{1, \mathrm{a}}$, Jingyi Han ${ }^{1, b^{*}}$ \\ ${ }^{1}$ School of Information and Control Engineering, Shenyang Jianzhu University, Shenyang 110168 , \\ China \\ a Limenxin@sjzu.edu.com, ${ }^{\mathrm{b}} 13840515221 @ 163 . c o m$
}

\begin{abstract}
In this paper, the statistical characteristics of complex networks are introduced, and basic four kinds of models of complex networks are analyzed. Based on the theory of complex network, it is carried out about the research on the three networks such as road network, bus network and track network in urban transportation system. The topological characteristics of the networks in urban transportation system are accordingly illustrated. The complex network theory for three kinds of network research is discussed according to the topological characteristics from dynamic analysis with two aspects. Finally, the research difficulties and direction are presented.
\end{abstract}

Keywords: complex network, road network, public transportation network, track network

\section{Introduction}

Complex systems are systems with a moderate number of intelligent, adaptive subjects based on local information. Complex network is the basic framework of complex system, through study on complex networks can better explain the structure and function of the system. Traffic system is a kind of complex system. Study the complexity of urban transport network and topology characteristics, for transportation planning is of great significance. At present, most of the research on traffic network is concentrated on the road, bus and track, and these studies can be divided into two categories: one is the study of the topology of the network; one is the dynamic analysis of the network.

\section{Complex Networks}

\subsection{Statistical Characteristics}

1) Degree and degree distribution

In the abstract graph, the number of other nodes connected to each node is defined as a degree, which is also a key attribute that determines the importance of the node. The degree distribution is the concept of graph theory and network theory, and it is one of the most important parameters to describe the complex characteristics of the network.

\section{2) Average path length}

The average path length is a more efficient measure of the topology. The distance between two nodes in the network is defined as the number of edges between the shortest paths connecting the two nodes. The maximum distance between any two nodes in the network is the diameter of the network. The average path length of the network is also called the characteristic path length of the network. It is the average of the distance between any two nodes.

\section{3) Aggregation factor}

The aggregate coefficient is a parameter that used to describe the degree of aggregation of nodes in a network graph, which can represent the intimate relationship between each node. The coefficient is the average of the ensemble coefficient of all nodes in the network.

\subsection{Complex Network Model}

1) Rules Network

A rule network is the simplest structure that uses nodes and connections to describe the links between things. In the regular network model, the node degree distribution obeys the $\delta$ distribution, and has a large aggregation coefficient and an average path length. 


\section{2 ) Random network}

Unlike the rule network, the node degree distribution of the random network conforms to the Poisson distribution. The ER stochastic model proposed by P. Erdos and A. Renyi [1] has a typical small-world feature which connects the $\mathrm{N}$ nodes in the network with the probability $\mathrm{p}$ to form a total of $\mathrm{P} * \mathrm{~N} *(\mathrm{~N}-1) / 2$ edge of the network model, its average path length to its scale for the logarithmic growth.

\section{3) Small World Network}

In 1998 Watts and Strogtz [2] proposed a new form network model, which is a small-world network model, also known as WS small-world model, as a new form network model between regular networks and random networks. WS network model [3] has a high clustering, but smaller average path length characteristics.

4) scale-free network

With the deep study of complex networks, people have proposed the scale-free network after the small world network. The degree distribution in the scale-free network is consistent with the power-law distribution. In this type of network, the degree of convergence of the nodes has no significant characteristic length. AL Barabasi and R Albert [4] proposed a classical scale-free model called BA model, and consider the real network growth characteristics and priority connectivity.

\section{An Empirical Study on Urban Traffic Network}

\subsection{Urban Road Network}

Urban road network is composed of different functions, grade, location of the road, with a certain density and the appropriate form of the network structure. Sergio Porta [5] analysis and found both with scale-free and small world characteristics, also proposed the MCA method [6] to conduct empirical studies on four real urban road systems.

There are two main modeling methods of urban road network, which are divided into original mapping method and dual method. The original mapping method abstracts the traffic path into the edge of the network model and abstracts the intersection of the traffic path into the node in the network model. The dual method is to abstract the intersection of the road as the edge of the topology network and abstract the road segment as the node of the topology network.

\subsection{City Bus Network}

Public transportation network as one of the main tools of energy saving, environmental protection and effective solution to traffic problems such as urban congestion, it can improve the efficiency of traffic by analyzing and optimizing it. Julian Sienkiewicz [7] Topological analysis of 22 urban bus networks in Poland. Seaton and Hackett [8] calculate the complex statistical characteristics of the city train network. Domestic research, including Qian Xu [9] on the city's public transport network degree of the distribution of the situation.

\subsection{Urban Rail Network}

In the context of urbanization to accelerate the construction and construction of low-carbon economy, people pay more and more attention on the construction of the track in our country. As one of the blood vessels of urban public transport, the track has the advantages of large passenger capacity, fast speed and punctuality. In a foreign study, M. Marchiori and V. Latora $[10,11]$ studied the Boston map and analyzed the characteristics from both the global and local levels. P.Sen [12] studied the small world of the Indian railway network Characteristics; Boston and Vienna between the two cities of the railway network analysis; Indian railway network proposed the construction of $\mathrm{P}$ space method, and in this space analysis of its small world characteristics.

Urban rail networks have linear growth and preference to attach two characteristics. The size of the urban rail network will change as new lines or sites grow, similar to public transportation networks. 


\section{Complex Network Theory of Urban Traffic Network}

Complex network theory of urban transportation network is mainly embodied in two aspects: one is the analysis of the characteristics of the traffic network topology, the second is based on the topology of the traffic network dynamics analysis.

\subsection{Urban Road Network}

1) Topological characteristics analysis

The topology of the road network has complex network characteristics, the average path length is small and the aggregation coefficient is large. In addition, the degree distribution is consistent with the power-law distribution, is a typical small-world network.

2) Network dynamics analysis

The reliability analysis of the road is divided into connectivity reliability, travel reliability and capacity reliability. Connectivity reliability analysis of road connectivity, travel reliability analysis can predict travel time, capacity reliability analysis can understand the city carrying passenger flow, traffic flow.

The road network topology model is established, and the robustness evaluation index is used to attack the road under the robustness evaluation strategy, and the robustness of the road network under the attack is analyzed.

Urban road network evolution is time and space complexity. With the change of time, the network will show different topological characteristics, different cities of the network structure because of traffic patterns, the development level and different. For the network topology evolution can be from the network fractal features, accessibility research, topology attributes of the three methods.

\subsection{Urban Bus Network}

1) Topological characteristics analysis

As a part of the transportation network, the network can be abstracted as a docking network model and a bus line network model composed of bus lines and stops. The number of stops in the public transportation network is very large, and as the new bus lines or sites appear, the overall network will change. In addition, the city bus network is planned, does not meet the complete random network.

2) Network dynamics analysis

One of the most important ways to reduce traffic congestion is to encourage travelers to use public transport networks. Analysis of the reliability of public transport network, improve the level of public transport network services have great social benefits.

The bus network model are constructed respectively, and the robustness of the urban public transport network is defined, and the robustness is observed under the attack strategy.

Evolution of the public transport network except for increasing the lines, such as between the two sites between the bus lines and sites, the evolution of the process may also occur to replace the site or the line between the sites.

\subsection{Urban Rail Network}

1) Topological characteristics analysis

For the rail network, the $\mathrm{L}$ space and the $\mathrm{P}$ space have different properties. In the $\mathrm{L}$ space, the degree distribution of the rail network is concentrated, the average path length is large, and the aggregation coefficient is small. In the P space, the network topology is opposite.

2) Network dynamics analysis

The reliability analysis of the rail network is mainly two forms: one is the reliability of connectivity; the other is operational reliability, including. In the case of simulation, the global efficiency of the network is established as the connectivity reliability of the urban rail transit network. Through the complex network knowledge analysis of operational reliability, to avoid external factors or facilities, such as interference, to ensure the normal operation of rail transit.

The robustness of the orbital network can accurately locate the key hub in the orbital network and is important for the traffic layout and the ability to improve the defensive capability of the rail transit. It also can meet the traffic demand in various periods and regions. 
The rail transit network belongs to the open system, and its evolution is accompanied by the gradual development of the urban spatial layout and the overall demand pattern, and has different characteristics of the development stage of the life cycle, from the early to the stationary period.

\section{Conclusion}

With the rapid development of complex network theory and widely used, not only solve the problems of many disciplines, but also improve transportation planning and management. Complex network research can be predicted and controlled network behavior by analyzing structures and functions. Based on the analysis of the complex network theory, the dynamic analysis of the network can be carried out on the basis of the analysis of the topology of the traffic network, and the small world or scale-free characteristics of the traffic network can be analyzed. And then the robustness to the network, reliability and evolution of many studies to be determined the network behavior. On the one hand, it is possible to put forward effective measures to prevent the attack of the network and solve the traffic problems such as traffic congestion and accidents. On the other hand, it is of practical significance to facilitate the traffic planning and traffic management.

\section{References}

[1]Erdos P., Renyi A., Erdos P., Publications of the Mathematical Institute of the Hungarian Academy of Sciences[J]. Graph Theory, 1956.

[2]Watts D J., The "New" Science of Networks [J]. Annual Review of Sociology, 2004, 30(5):243-270.

[3]Bo W., Wanliang W., Xuhua Y., Study on Modeling and Simulation of WS and NW Small World Network Models [J]. Journal of Zhejiang University of Technology, 2009, 37 (2) 179-182.

[4]Albert R E., Barabasi A L., Statistical mechanics of complex networks. Reviews of Modern Physica, 2002, 74, 47-97.

[5]Porta S., Crucitti P. and Latora V., The network analysis of urban streets: a dual approach [J] .Physica A: Statistical Mechanics and its Applications, 2006, 369 (2) 853-866.

[6]Porta S., Crucitti P., Latora V. The network analysis of urban streets: a dual approach [J]. Environment and Planning B: Planning and Design, 2006, 369(5) 853-866.

[7]Sienkiewicz J., Hołyst J A., Statistical analysis of 22 public transport networks in Poland[J]. Physical Review E Statistical Nonlinear \& Soft Matter Physics, 2005, 72(2) 046-127.

[8]Seaton K A., Hackett L M., Stations, trains and small-world networks[J]. Physica A Statistical Mechanics \& Its Applications, 2003, 339(3-4) 635-644.

[9]Qian X., Zhenghu Z., Zhijing X., Wendou Z., Tao Z., 330 Chinese cities P space under the bus complex network empirical study [J]. Journal of Transportation Systems Engineering and Information, 2013, (1) 193-198.

[10]Latora V., Marchiori M., Is the Boston subway a small-world networks[J]. Physica A Statistical Mechanics \& Its Applications, 2002, 314(1-4) 109-113.

[11]Latora V., Marchiori M., Economic small-world behavior in weighted networks [J]. The European Physical Journal B, 2003, 32,249-263.

[12] Sen P., Dasgupta S., Chatterjee A., Small-world properties of the Indian railway network [J]. Physical Review E Statistical Nonlinear \&Soft Matter Physics, 2003, 67(3) 036-106. 